\title{
Simplified surgical approach to improve surgical outcomes in the center with a small volume of acute type A aortic dissection surgery
}

\author{
Jong Hun Kim ${ }^{\mathrm{a}, \mathrm{b}, \mathrm{c}, 1}$, Jong Bum Choi ${ }^{\mathrm{a}, \mathrm{b}, \mathrm{c}, 1, *}$, Tae Youn Kim ${ }^{\mathrm{a}, 1}$, Kyung Hwa Kim ${ }^{\mathrm{a}, 1}$ and \\ Ja Hong Kuh ${ }^{\mathrm{a}, 1}$ \\ ${ }^{a}$ Department of Thoracic and Cardiovascular Surgery, Chonbuk National University Medical School, \\ Jeonju, Chonbuk, Korea \\ ${ }^{\mathrm{b}}$ Research Institute of Clinical Medicine of Chonbuk National University, Jeonju, Chonbuk, Korea \\ ${ }^{\mathrm{c}}$ Biomedical Research Institute of Chonbuk National University Hospital, Jeonju, Chonbuk, Korea
}

Received 25 December 2017

Accepted 22 May 2018

\begin{abstract}
.
BACKGROUND: Despite recent advances in surgical techniques and perioperative management, the surgical mortality of acute type A aortic dissection remains high.

OBJECTIVE: In a hospital with few acute type A aortic dissection operations, we retrospectively investigated whether simplified surgical approach could obtain proper surgical outcomes.

METHODS: Between October 2007 and December 2016, we performed emergency surgery in 99 patients who had acute type A aortic dissection, including replacement of the hemi-arch in 62 patients $(63 \%)$ and total arch in 32 patients (32\%) and aortic root surgery in 7 patients (7\%). Surgical strategy has been simplified over time.

RESULTS: We performed right axillary artery perfusion in 61 patients $(62 \%)$ and antegrade cerebral perfusion in 78 patients (79\%). During the last 3 years, in-hospital mortality was decreased to $4 \%(2 / 47)$. Preoperative unresolved shock was an independent predictor of hospital death. Although the patients with total arch replacement or aortic root surgery had a mean significantly long cardiopulmonary bypass or circulatory arrest time, in-hospital mortality or neurologic complications was not increased.
\end{abstract}

CONCLUSIONS: Simplified surgical approach could provide a reasonable surgical outcome in acute type A aortic dissection surgery in a center with a small volume of acute aortic dissection surgery.

Keywords: Aorta, acute dissection, surgery, treatment outcome

\footnotetext{
${ }^{1}$ These authors contributed equally to this work.

${ }^{*}$ Corresponding author: Jong Bum Choi, Department of Thoracic and Cardiovascular Surgery, Chonbuk National University Medical School, 20 Geonji-Ro, Deokjin-Gu, Jeonju 54907, Chonbuk, Korea. Tel.: +82 63859 2545; Fax: +82 632501480 ; E-mail: jobchoi@jbnu.ac.kr.
}

0928-7329/18/\$35.00 (c) 2018 - IOS Press and the authors. All rights reserved

This article is published online with Open Access and distributed under the terms of the Creative Commons Attribution NonCommercial License (CC BY-NC 4.0). 


\section{Introduction}

Despite progressively advanced surgical strategy and techniques, surgery for acute type A aortic dissection still has high hospital mortality (12\%-22\%) and low 5-year survival (59\%-66\%) [1-4] including high incidence of postoperative stroke (10\%) [2-5].

Recently antegrade cerebral perfusion for brain protection during circulatory arrest has significantly reduced in-hospital mortality after acute type A aortic dissection surgery [6].

The purpose of this retrospective study was to investigate surgical outcomes associated with simplified surgical approach for acute type A aortic dissection surgery in a center with small number of acute aortic type A dissection operations.

\section{Methods}

\subsection{Patients and preoperative characteristics}

From October 2007 to December 2016, 99 consecutive patients (50 men and 49 women; mean age, 59 $\pm 14 \mathrm{y}$ ) had emergency surgery (within 3 hours after the arrival at the emergency department) for repair of acute type A aortic dissection (Table 1). In patients suspected of acute dissection from symptoms and signs, acute aortic dissection was diagnosed by contrast computed tomography (CT) angiography, and myocardial dysfunction, pericardial tamponade, extension of aortic dissection into the aortic sinuses, and heart valve function were evaluated by transthoracic echocardiography. Most patients arrived at the emergency department within 24 hours after onset of symptoms. Preoperative characteristics of the patients are listed in Table 1.

\subsection{Surgical procedure}

Perioperative findings are listed in Table 2. Surgical approach was simplified with time and nearly unified by the end of 2013, as listed in Table 3. Preoperative unresolved shock, which was defined as an unstable preoperative blood pressure less than $90 \mathrm{mmHg}$ with inotropic support and volume replacement, was moved to the operating room immediately after the diagnosis.

Surgery was performed through median sternotomy. Intraoperatively arterial pressure monitoring was performed at three sites of both radial arteries and the left dorsalis pedis artery or left femoral artery. In hemodynamically stable patients, arterial perfusion was achieved through right axillary artery using an 8-mm graft. For retrograde cardioplegia through the purse-string sutured coronary sinus aperture, a small right atriotomy was made with 2 vena cava cannulations. For myocardial protection, retrograde cardioplegia was performed at 20-30-minute intervals. Circulatory arrest for hemi-arch replacement (HAR) or total arch replacement (TAR) was performed at $20^{\circ} \mathrm{C}$ bladder temperature.

During cooling, the middle portion of the ascending aorta was clamped and opened transversely to locate the intimal tear. Supra-coronary transection was performed to examine the extent of dissection in the sinuses, functional morphology of the aortic valve, and injuries of the coronary ostia. In the early study period, the dissected aortic root was repaired with felt pseudomedia (Teflon felt, Bard PTFE, IMPRA, Tempe, AZ, USA) after resuspension of the aortic commissures [7]; in the latter period, the dissected aortic root was repaired with BioGlu (CryoLife, Inc., Kennesaw, GA, USA) and the distal end was reinforced with double felt strips [8]. 
Table 1

Preoperative characteristics of patients who had surgery for acute type A aortic dissection*

\begin{tabular}{|c|c|}
\hline Characteristic & No. of patients $(\%)$ \\
\hline Male sex & $50(51)$ \\
\hline Arrival at emergency department within $24 \mathrm{hr}$ after symptom onset & $90(91)$ \\
\hline Smoking (current or previous) & $19(19)$ \\
\hline Hypertension & $68(69)$ \\
\hline Diabetes mellitus & $5(5)$ \\
\hline Chronic pulmonary disease & $5(5)$ \\
\hline Central neurologic deficit ${ }^{\mathrm{a}}$ & $9(9)$ \\
\hline Renal dysfunction $^{\mathrm{b}}$ & $5(5)$ \\
\hline Marfan syndrome & $4(4)$ \\
\hline Ventricular dysfunction ${ }^{\mathrm{c}}$ & $5(5)$ \\
\hline Aortic regurgitation $\geqslant$ moderate & $43(43)$ \\
\hline Malperfusion $^{\mathrm{d}}$ & $19(19)$ \\
\hline Shock (uncorrected hypotension) & $32(32)$ \\
\hline Hemopericardium & $38(38)$ \\
\hline
\end{tabular}

${ }^{*} N=99$ patients. Data reported as number of patients $(\%) .{ }^{\mathrm{a}}$ History of transient ischemic attack or stroke. ${ }^{\mathrm{b}}$ Preoperative serum creatinine level $>2.0 \mathrm{mg} / \mathrm{dL}$. ${ }^{\mathrm{c}}$ Left ventricular ejection fraction $<50 \%$. ${ }^{\mathrm{d}}$ Severe stenosis of cerebral artery, visceral artery, or artery below the bifurcation of the aorta.

Table 2

Perioperative findings and operative data in patients who had surgery for acute type A aortic dissection*

\begin{tabular}{lc}
\hline Findings & Data \\
\hline Cardiopulmonary bypass time (min) & $176 \pm 71$ \\
Hypothermic circulatory arrest time (min) & $59 \pm 35$ \\
Antegrade cerebral perfusion time (min) & $60 \pm 34$ \\
Retrograde cerebral perfusion time (min) & $52 \pm 39$ \\
Arterial perfusion & $61(62)$ \\
$\quad$ Right axillary artery, 8-mm artificial graft & $38(38)$ \\
Right femoral artery, straight cannula & \\
Aortic valve regurgitation & $39(39)$ \\
None or trivial & $17(17)$ \\
Mild & $25(25)$ \\
Moderate & $18(18)$ \\
Severe & $7(7)$ \\
Annuloaortic ectasia (5 cm > root dimensions) & \\
Intimal tear & $40(40)$ \\
Ascending aorta & $39(39)$ \\
Aortic arch & $10(10)$ \\
Aortic root (sinus) & $10(10)$ \\
Unidentified & \\
Cerebral perfusion during circulatory arrest & $78(79)$ \\
Antegrade cerebral perfusion & $16(16)$ \\
Retrograde cerebral perfusion & $5(5)$ \\
No circulatory arrest & \\
Sinus impending rupture (with bleeding) & $6(6)$ \\
Aortic root replacement or reimplant & $7(7)$ \\
Extents of resection & $62(63)$ \\
Hemiarch & $32(32)$ \\
Total arch & $5(5)$ \\
Ascending aorta &
\end{tabular}

${ }^{*} N=99$ patients. Data reported as mean $\pm \mathrm{SD}$ or number of patients $(\%) .{ }^{\dagger}$ Ascending aorta only was replaced under moderate hypothermia. 
Table 3

Simplified surgical approach for acute type A aortic dissection surgery

\begin{tabular}{lll}
\hline Variables & The early period & The latter period \\
\hline Cardioplegia & Antegrade & Retrograde \\
Dissected aortic sinuses & Pseudomedia (Teflon felt) & BioGlue \\
Dissected aortic walls & \multicolumn{2}{c}{ Sandwitched with two Teflon strips } \\
Cerebral perfusion & Retrograde & Antegrade \\
Systemic hypothermia & \multicolumn{3}{c}{$20^{\circ} \mathrm{C}$} & \\
Head vessel anastomosis & \multicolumn{3}{c}{ Carrol patch } \\
\hline
\end{tabular}

After systemic circulation was arrested, the ascending aorta was unclamped and the intima and false lumen in the aortic arch were examined. The primary intimal tear was identified in 89 patients (90\%) (Table 2). For hemi-arch replacement (HAR) or total arch replacement (TAR), 78 patients (79\%) had bilateral selective antegrade cerebral perfusion and 16 patients $(16 \%)$ in the early period had retrograde cerebral perfusion. HAR were performed in 67 patients (68\%), and TAR in 32 patients (32\%). TAR was indicated in cases with intimal tear near head vessels, primary entry located at descending aorta or unknown, or the need for total arch reconstruction dependent on patient age. For HAR, resection of the aortic arch was extended near the subclavian artery and ligamentum arteriosum [7]. The distal aorta was reinforced with double felt strips using a continuous 4-0 polypropylene mattress suture [8] and open distal anastomosis was performed with graft-in-aorta telescopic suture technique using a continuous 3-0 polypropylene suture.

In TAR procedure, the three head vessels were mobilized as a Carrel patch [10] and the brim of the patch was reinforced with double felt strips [8]. In 4 patients who had intima disruption of the innominate or left subclavian artery, a modified Carrel patch technique with a separate subclavian artery anastomosis was used [10]. A 4-branch graft was used in 2 patients who had intimal disruption in both innominate and left carotid arteries. After end-to-side anastomosis of the head vessel patch to the graft were performed, systemic antegrade perfusion was restarted through the right axillary artery and a side branch of the graft. The obliquely trimmed proximal end of the graft was anastomosed to the new sino-tubular junction. In all cases, we used a single vascular graft for HAR or TAR.

For treatment of aortic root dilation, 2 patients had aortic root reimplantation (David procedure) [11] and 5 patients had aortic root replacement (modified Bentall procedure) [12]. In 14 patients (14\%) who had sinus intimal tear, sinus resection and graft replacement $(n=5)$, sinus plication sutures $(n=5)$, and sinus patch reinforcement $(n=4)$ were performed after repair of the dissected aortic root using BioGlue.

\subsection{Simplified surgical approach for antegrade systemic perfusion and myocardial and brain protection}

Arterial perfusion for cardiopulmonary bypass was performed through right axillary artery in most patients with stable hemodynamics, and another arterial perfusion through a side branch of the aortic graft was added after arch repair. In patients who had initial femoral artery perfusion, after distal aortic anastomosis and arch vessel anastomosis, the arterial perfusion was converted to antegrade systemic perfusion through a side branch of the aortic graft. During the past decade, the surgical procedure has been gradually modified based on common evidences from many documents for the improvement of surgical outcomes. In the last three years, the surgical protocol was the same in most patients, except for some patients who required additional or modified surgery, as described in Table 3. In most patients who had total arch replacement, Carrel or modified Carrel patch technique was used for repair of the 
Table 4

Postoperative findings and outcomes in patients who had surgery for acute type A aortic dissection*

\begin{tabular}{lc}
\hline Parameter & Results \\
\hline Re-exploration due to bleeding & $4(4)$ \\
Ventilator support time $(\mathrm{h})$ & $92 \pm 85$ \\
Long ventilator support $(>72 \mathrm{~h})$ & $38(38)$ \\
Postoperative intensive care unit stay $(\mathrm{d})$ & $8 \pm 6$ \\
Delayed sternal closure & $10(10)$ \\
New stroke & $3(3)$ \\
Temporary neurologic deficit & $4(4)$ \\
Abdominal organ ischemic injury & $2(2)$ \\
Hospital stay (d) & $24 \pm 12$ \\
Early death (<24 h after surgery) & $5(5)$ \\
Hospital death, including early death & $8(8)$ \\
Late death & $3(3)$ \\
Clinical follow-up (mo) & $50 \pm 36$ \\
\hline
\end{tabular}

${ }^{*} N=99$ patients. Data reported as number of patients $(\%)$ or mean $\pm \mathrm{SD}$.

head vessels [10]. For myocardial protection, retrograde cardioplegia was performed through a 12-Fr balloon catheter directly inserted into coronary sinus with a purse-string suture at the aperture. For brain protection during circulatory arrest, bilateral selective antegrade cerebral perfusion was performed through the axillary artery or the innominate artery and the left carotid artery with balloon occlusion of the left subclavian artery. A left ventricular vent catheter was routinely placed through the right superior pulmonary vein.

\subsection{Statistical analysis}

All data analyses were performed with statistical software (SPSS, version 18.0, SPSS Inc, Chicago, IL, USA). Continuous variables are reported as mean \pm standard deviation and compared with MannWhitney test. Categorical data were compared with chi-square or Fisher exact test. Logistic regression on significant perioperative variables was performed to determine independent risk factors for hospital mortality. Kaplan-Meier curves were constructed to estimate survival. Statistical significance was defined by $P \leqslant 0.05$.

\section{Results}

The mean cardiopulmonary bypass time was $234 \pm 86 \mathrm{~min}$, including the mean cerebral perfusion time of $60 \pm 36$ min under hypothermic circulatory arrest, and the mean ischemic heart time was $162 \pm$ $59 \mathrm{~min}$. The mean lowest nasopharyngeal temperature was $15.2 \pm 2.5^{\circ} \mathrm{C}$ (Table 2). In-hospital mortality was $8 \%(8 / 99)$ in all cases; $12 \%(6 / 52)$ during the early 6 years and $4 \%(2 / 47)$ during the last 3 years that had establishment of the simplified surgical strategy $(P=0.27)$. The causes of the in-hospital death were myocardial infarction or arrhythmia due to coronary ostial injury associated with aortic sinus dissection in 4 patients, uncontrolled bleeding in 2 patients, and mesenteric infarction in 2 patients. Of them, 6 were died within 24 hours after surgery.

Postoperative complications included new stroke in 3 patients (3\%), aggravation of previous stroke in 3 patients (3\%), and prolonged ventilation $>72$ hours in 38 patients (38\%) (Table 4). The frequency of postoperative stroke was not different between retrograde and antegrade cerebral perfusions $(2 / 16,13 \%$ vs $4 / 78,5 \% ; P=0.28)$. There was no paraplegia secondary to spinal cord injury. Postoperative contrast 
Table 5

Univariate and multivariate analysis of hospital mortality in patients who had surgery for acute type A aortic dissection*

\begin{tabular}{|c|c|c|c|c|}
\hline Parameter & No. of patients (\%) & $\begin{array}{l}\text { No. of patients who } \\
\text { died in Hospital (\%) }\end{array}$ & $\begin{array}{l}\text { Univariate } \\
(P \text { value })\end{array}$ & $\begin{array}{l}\text { Multivariate } \\
(P \text { value })\end{array}$ \\
\hline Age (years) & & & 0.31 & \\
\hline$\leqslant 50$ & $33(33)$ & $2(6)$ & & \\
\hline $51-70$ & $36(36)$ & $5(14)$ & & \\
\hline$\geqslant 71$ & $30(30)$ & $1(3)$ & & \\
\hline Year range of surgery & & & 0.27 & \\
\hline $2007-2013$ & $52(53)$ & $6(12)$ & & \\
\hline 2014-2016 & $47(47)$ & $2(4)$ & & \\
\hline Male & $50(51)$ & $4(8)$ & 1.00 & \\
\hline Hypertension & $68(69)$ & $6(9)$ & 1.00 & \\
\hline Smoking & $19(19)$ & $2(11)$ & 0.65 & \\
\hline Diabetes mellitus & $5(5)$ & $0(0)$ & 1.00 & \\
\hline COPD & $5(5)$ & $0(0)$ & 0.35 & \\
\hline Stroke & $9(9)$ & $1(10)$ & 0.55 & \\
\hline Renal dysfunction $>$ stage 3 & $5(5)$ & $0(0)$ & 1.00 & \\
\hline \multicolumn{5}{|l|}{ Before surgery } \\
\hline $\mathrm{AR}>$ moderate & $18(18)$ & $1(6)$ & 1.00 & \\
\hline Shock (full inotropics) & $32(32)$ & $6(19)$ & 0.01 & 0.013 \\
\hline Hemopericardium & $38(38)$ & $5(13)$ & 0.25 & \\
\hline Iliac artery involvement & $51(52)$ & $8(16)$ & 0.01 & 0.99 \\
\hline Malperfusion & $19(19)$ & $4(21)$ & 0.04 & 0.14 \\
\hline \multicolumn{5}{|l|}{ Surgery } \\
\hline Arch intima tear & $40(40)$ & $5(13)$ & 0.26 & \\
\hline Retrograde cerebral perfusion & $16(16)$ & $4(25)$ & 0.03 & 0.13 \\
\hline All sinus dissection & $11(11)$ & $3(27)$ & 0.04 & 0.63 \\
\hline Total arch resection & $32(32)$ & $3(9)$ & 1.00 & \\
\hline Femoral artery cannulation & $38(38)$ & $6(16)$ & 0.06 & \\
\hline Sinus repair & $14(14)$ & $3(21)$ & 0.09 & \\
\hline Aortic root surgery & $7(7)$ & $0(0)$ & 1.00 & \\
\hline CPB time (minutes) & & & 0.01 & 0.28 \\
\hline$\leqslant 120$ & $3(3)$ & $0(0)$ & & \\
\hline $121-180$ & $68(69)$ & $3(4)$ & & \\
\hline $180-240$ & $18(18)$ & $1(6)$ & & \\
\hline$\geqslant 240$ & $10(10)$ & $4(40)$ & & \\
\hline HCA time (minutes) & & & 0.39 & \\
\hline$\leqslant 30$ & $15(15)$ & $2(13)$ & & \\
\hline $31-60$ & $45(45)$ & $2(4)$ & & \\
\hline$\geqslant 61$ & $33(33)$ & $3(9)$ & & \\
\hline \multicolumn{5}{|l|}{ After surgery } \\
\hline Delayed sternal closure & $10(10)$ & $1(10)$ & 1.00 & \\
\hline Ventilation time $>72$ hours & $38(38)$ & $2(4)$ & 1.00 & \\
\hline ICU stay $>5$ days & $41(41)$ & $3(7)$ & 0.09 & \\
\hline
\end{tabular}

${ }^{*} N=99$ patients. Data reported as number of patients (\%). AR: aortic valve regurgitation; COPD: chronic obstructive pulmonary disease; CPB: cardiopulmonary bypass; HCA: hypothermic circulatory arrest; ICU: intensive care unit. $P<0.05$ was considered statically significant.

CT angiography was performed in 95\% (86/91 survivors) and revealed that 56\% (48/86 examiners) had false lumen thrombosis.

Univariate analyses showed that predictive factors for hospital death included preoperative shock, iliac artery involvement, poor preoperative perfusion, retrograde cerebral perfusion, all sinuses' dissection, and longer cardiopulmonary bypass time (Table 4). Multivariate analysis showed that preoperative shock was an independent predictor of hospital death (relative risk, 9.3; 95\% confidence interval 1.6-53.6; $P=$ 0.01) (Table 5). 


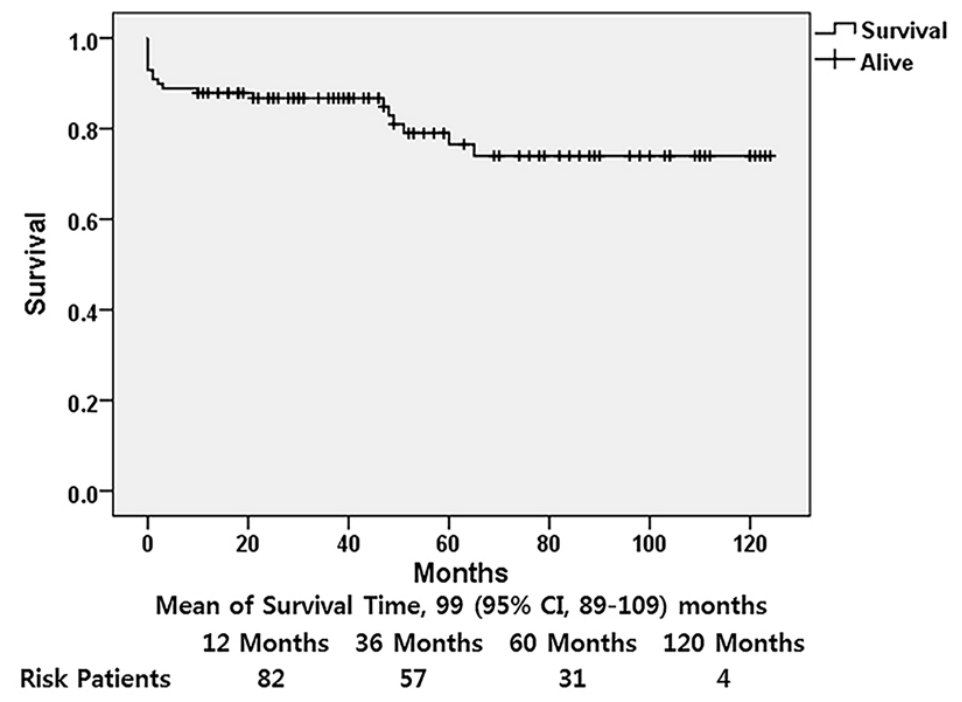

Fig. 1. Kaplan-Meier curve showing survival after surgery for acute type A aortic dissection.

Between the patients who had HAR and TAR, the TAR group was significantly younger than the HAR group $(53 \pm 11$ y vs $61 \pm 14 \mathrm{yr} ; P<0.01)$. Although the TAR group had significantly longer hypothermic circulatory arrest time than the HAR group ( $92 \pm 41$ min vs $43 \pm 15$ minutes; $P<0.001)$, TAR was not a risk factor for operative death (TAR, $3 / 32$ vs HAR, $5 / 62 ; P=1.00$ ) and the incidences of stroke were similar for the 2 procedures (HAR, $5 / 62$ vs TAR, 1/32; $P=0.75$ ). Patients with additional aortic root surgery ( 5 replacement or 2 reimplantation) had significantly longer bypass time than those without aortic root surgery ( $299 \pm 130 \mathrm{~min}$ vs. $167 \pm 56 \mathrm{~min}, P=0.04)$, but the aortic root surgery was not a risk factor of hospital death $(0 / 7$ vs $8 / 92 ; P=1.00)$. Of the patients with aortic root surgery, 2 patients had a coronary occlusion of the right or left coronary artery caused by ostial intima disruption, respectively. Both received interposition of a short vein graft between the trimmed proximal coronary artery and the replaced aortic root.

Between patients that had undergone retrograde and antegrade cerebral perfusions, patients in the two perfusion groups had a similar mean circulatory arrest time (antegrade, $60 \pm 34$ min: retrograde, $52 \pm$ 39 min; $P=0.25$ ), but the retrograde perfusion group had longer ventilation support compared with the antegrade group $(146 \pm 125 \mathrm{~h}$ vs $74 \pm 69 \mathrm{~h} ; P=0.004)$.

The median follow-up time was 44 months (range, 4-124 m; mean, $50 \pm 36 \mathrm{~m}$ ), with a complete follow-up of 91 survivors. In all patients, the survival rates at 1 and 5 years after surgery were $88 \%$ and $77 \%$, respectively (Fig. 1).

After the first surgery, 3 patients (3\%) had aortic reinterventions: 1 patient had resection of a noncoronary sinus aneurysm and graft replacement at 35 months after the first operation, and 2 patients had resection of thoracoabdominal aortic aneurysm at 19 months and 24 months each after the dissection surgery and survived.

\section{Discussion}

During the 9 years of surgery for aortic dissection, we have tried to unify the surgical protocol as one of the efforts to obtain a low surgical mortality in our institute that had a small volume of acute 
type A aortic dissection operations. Our patient cohort had an overall in-hospital mortality of $8 \%$. In the 3 years since January 2014, in-hospital mortality decreased to $4 \%$, which is similar to the results of a recent study [13]. These improved outcomes may be secondary to simplified surgical strategy, such as right axillary artery perfusion, sufficient excision of dissected aortic wall, proper approximation of the remaining dissected aortic walls, bilateral selective antegrade cerebral perfusion during circulatory arrest, hemostatic anastomosis, and appropriate use of Carrel or modified Carrel patch technique.

Surgical centers participating in a small volume of aortic dissection operations may show high mortality because of insufficient surgical experiences. During our experience of 9 years, surgical strategy had been unified to improve surgical outcome. The simplified surgical approach has made surgeons more familiar with the operative procedures of acute aortic dissection. Application of different surgical protocols to every case cannot predict the surgical outcome.

We performed ascending aorta clamping and aortic root repair during cooling, confirming absence of malperfusion by monitoring blood pressure in 3 different arterial cannulation sites. With aortic crossclamping in the middle level of the ascending aorta, we did not see malperfusion sign, even in patients who had the entry site in the ascending aorta.

According to data from the International Registry of Acute Aortic Dissection, independent variables associated with death include advanced age, hypotension, shock, previous heart disease, and preoperative renal, mesenteric or myocardial ischemia [1]. In our study, preoperative unresolved shock was the only independent risk factor of hospital death, but advanced age was not a risk factor. Recently even in octogenarians, surgical outcome was favorable [14].

Total arch replacement for acute aortic dissection was reported to cause hospital mortality, and the main causes of death were neurologic deficits and low cardiac output [2,4]. The causes of death may be related to inadequate cerebral perfusion and myocardial protection during cardiopulmonary bypass and circulatory arrest. The incidence of temporary and permanent neurologic deficits has been reduced by antegrade cerebral perfusion $[15,16]$. Our brain protection method was bilateral selective antegrade cerebral perfusion. The neurologic complications could be reduced by deep hypothermia and integrated antegrade cerebral perfusion during circulatory arrest $[15,17]$. Recently, moderate hypothermia $\left(23^{\circ} \mathrm{C}\right)$ has showed less operative mortality and neurologic complications than deep hypothermia $\left(17^{\circ} \mathrm{C}\right)$ in emergent and elective aortic arch surgery [18]. In another study, mild hypothermia $\left(28-30^{\circ} \mathrm{C}\right)$ and antegrade cold blood cerebral perfusion $\left(20^{\circ} \mathrm{C}\right)$ showed low operative mortality without neurologic complications [19]. We used deep hypothermia, but the addition of the antegrade or retrograde cerebral perfusion showed low neurologic deficit, except delayed wakening in patients who had retrograde cerebral perfusion. In future, we should also consider the proper use of mild to moderate hypothermia. Until November 2013, we frequently used retrograde cerebral perfusion with a mean circulatory arrest time of 52 minutes. Retrograde cerebral perfusion did not affect neurologic deficits and hospital mortality rates, but delayed awakening of the patients caused prolonged mechanical ventilation time. Retrograde cerebral perfusion may be safe in the circulatory arrest time $<45 \mathrm{~min}$ [20], but in cases that require longer circulatory arrest, antegrade cerebral perfusion should be considered. We thought the unique disadvantage of deep hypothermia associated with cerebral perfusion is extended cardiopulmonary bypass time due to cooling and rewarming times.

Postoperative neurologic complications may be associated with retrograde arterial perfusion from the femoral artery $[15,16]$. Compared with femoral artery cannulation for arterial perfusion, right axillary artery cannulation reduced the risk of poor visceral and cerebral perfusion and embolism from the thoraco-abdominal aorta to the brain [21,22]. Our practice for arterial perfusion in acute aortic dissection surgery is right axillary artery perfusion using an $8-\mathrm{mm}$ vascular graft. However, in patients who have 
unresolved hypotension, right femoral artery cannulation is inevitable for emergency cardiopulmonary bypass. In these patients, after distal aortic anastomosis of the graft under antegrade cerebral perfusion, we converted the femoral artery perfusion to the antegrade systemic perfusion through a side branch of the aortic graft.

Myocardial protection during cardiac arrest is an important tool to prevent postoperative low cardiac output. The longer the operation time, the more emphasis is placed on myocardial protection [23]. In all cases, we performed retrograde cardioplegia every 20-30 minutes through a 12-Fr catheter, which was directly placed in coronary sinus with a purse-string suture through a short right atriotomy. This method does not require operative interruption for the infusion of cardioplegic solution, and it can induce perfusion of the entire myocardium through the middle cardiac vein by positioning the cannula balloon at the aperture of coronary sinus as possible [24].

In patients who had aortic regurgitation combined with aortic root dilation, aortic root reimplantation or replacement can be aggressively performed according to the pathology of the aortic valve during the initial procedure $[13,25]$. Aortic root surgery associated with acute type A aortic dissection surgery did not affect surgical outcomes of acute dissection surgery [13].

We had aortic reintervention in 3 patients only: resection of a sinus aneurysm in 1 patient and resection of descending thoracic aortic aneurysm in 2 patients. Such a low reintervention rate may be due to sufficient resection of the dissected aortic tissues and proper approximation of the dissected aortic walls. In TAR for aortic dissections extending to the thoracoabdominal aorta, we did not use elephant trunk technique because it was difficult to insert the elephant graft into the unexpanded intima of the aortic dissection. Instead, a graft length of $3 \mathrm{~cm}$ between the left subclavian artery and the distal aortic anastomotic suture line was left as a clamping site for future descending aortic operations.

Survival rates after surgery are similar in most reports [5,26,27], and risk factors for long-term survival included preoperative diabetes, advanced age, and postoperative dialysis [27]. However, our study population had a high survival curve after discharge, probably due to more younger patients in our study and low incidences of risk factors of late death.

Limitations of the present study included retrospective and nonrandomized design and a small number of the study cases. The small sample size and low mortality and morbidity rates can limit the statistical power for evaluating risk factors for events. Because the various parts of the surgical protocol have been simplified step by step at different times, it is difficult to determine an exact time point to compare early and late surgical outcomes. Although our surgical strategy was not modernized, including levels of hypothermia, we tried to perform the acute dissection surgery with reasonable surgical protocol. We achieved reasonable surgical outcomes with deep hypothermia, but recently the improved results were reported with mild to moderate hypothermia, which we need further studies about.

\section{Conclusion}

Emergent surgery for acute type A aortic dissection yielded acceptable early and intermediate results. Preoperatively uncorrected severe hypotension was an independent risk factor of in-hospital death in acute aortic type A dissection surgery. The causes of postoperative mortality or morbidity could be reduced with improved brain and myocardial protection. Bilateral selective antegrade cerebral perfusion is a reliable way to protect the brain during circulatory arrest. Our surgical strategy, including antegrade systemic arterial perfusion, effective myocardial protection, deep hypothermia and antegrade bilateral cerebral perfusion has been gradually simplified over a period of 9 years. With the simplified surgical approach, we could obtain satisfactory surgical outcomes in a hospital with a small number of type A 
aortic dissection operations. The majority of in-hospital deaths occurred because of preoperative acute myocardial injury and mesenteric ischemia, which were not related to surgical procedures during the last 3 years.

\section{Acknowledgments}

This study was supported by funds from the Research Institute of Clinical Medicine of Chonbuk National University and the Biomedical Research Institute of Chonbuk National University Hospital.

\section{Conflict of interest}

None to report.

\section{References}

[1] Rampoldi V, Trimarchi S, Eagle KA, Nienaber CA, Oh JK, Bossone E, et al. Simple risk models to predict surgical mortality in acute type A aortic dissection: The International Registry of Acute Aortic Dissection score. Ann Thorac Surg 2007; 83(1): 55-61.

[2] Kim JB, Chung CH, Moon DH, Ha GJ, Lee TY, Jung SH, et al. Total arch repair versus hemiarch repair in the management of acute DeBakey type I aortic dissection. Eur J Cardiothorac Surg 2011; 40(4): 881-7.

[3] Shimizu K, Ito M, Kanetsuki K, Imai K, Suehiro S, Oda T. Influence of an operative strategy on outcomes of acute type A aortic dissection cases. Kyobu Geka 2016; 69(4): 252-6.

[4] Lio A, Nicolò F, Bovio E, Serrao A, Zeitani J, Scafuri A, et al. Total arch versus hemiarch replacement for type A acute aortic dissection: A single-center experience. Tex Heart Inst J 2016; 43(6): 488-95.

[5] Ohara T, Koga M, Tokuda N, Tanaka E, Yokoyama H, Minatoya K, et al. Rapid identification of type A aortic dissection as a cause of acute ischemic stroke. J Stroke Cerebrovasc Dis 2016; 25(8): 1901-6.

[6] Perreas K, Samanidis G, Thanopoulos A, Georgiopoulos G, Antoniou T, Khoury M, Michalis A, et al. Antegrade or retrograde cerebral perfusion in ascending aorta and hemiarch surgery? A propensity-matched analysis. Ann Thorac Surg 2016; 101(1): 146-52.

[7] Kaiser LR, Kron IL, Spray TL. Mastery of Cardiothoracic Surgery. 3rd ed. Philadelphia: Wolters Kluwer; 2014. p. 1210.

[8] Kouchoukos NT, Blackstone EH, Hanley FL, Kirklin JK. Acute aortic dissection. in: Kirklin/Barratt-Boyes Cardiac Surgery. Philadelphia (PA): Elsevier; 2013. p. 941.

[9] Oda T, Minatoya K, Sasaki H, Tanaka H, Seike Y, Itonaga T, et al. Adventitial inversion technique for type A aortic dissection distal anastomosis. J Thorac Cardiovasc Surg 2016; 151(5): 1340-5.

[10] Elefteriades JA. Acute dissection. In: Coselli JS, LeMaire SA. Aortic Arch Surgery. Hoboken (NJ): Wiley-Blackwell; 2008. p. 258.

[11] David TE. Aortic valve sparing operations: A review. Korean J Thorac Cardiovasc Surg 2012; 45(4): 205-12.

[12] Michielon G, Salvador L, Da Col U, Valfrè C. Modified button-Bentall operation for aortic root replacement: The miniskirt technique. Ann Thorac Surg 2001; 72(3): S1059-64.

[13] Kunihara T, Neumann N, Kriechbaum SD, Aicher D, Schäfers HJ. Aortic root remodeling leads to good valve stability in acute aortic dissection and preexistent root dilatation. J Thorac Cardiovasc Surg 2016; 152(2): 430-6.

[14] Omura A, Matsuda H, Minami H, Nakai H, Henmi S, Murakami H, et al. Early and late outcomes of operation for acute type A aortic dissection in patients aged 80 years and older. Ann Thorac Surg 2017; 103(1): 131-8.

[15] Cabasa A, Pochettino A. Surgical management and outcomes of type A dissection-the Mayo Clinic experience. Ann Cardiothorac Surg 2016; 5(4): 296-309.

[16] Yamashita K, Kazui T, Terada H, Washiyama N, Suzuki K, Bashar AH. Cerebral oxygenation monitoring for total arch replacement using selective cerebral perfusion. Ann Thorac Surg 2001; 72(2): 503-8.

[17] Takahara Y, Mogi K, Sakurai M, Nishida H. Total aortic arch grafting via median sternotomy using integrated antegrade cerebral perfusion. Ann Thorac Surg 2003; 76(5): 1485-9.

[18] Tsai JY, Pan W, Lemaire SA, Pisklak P, Lee VV, Bracey AW, et al. Moderate hypothermia during aortic arch surgery is associated with reduced risk of early mortality. J Thorac Cardiovasc Surg 2013; 146(3): 662-7. 
[19] Suzuki T, Asai T, Nota H, Kuroyanagi S, Kinoshita T, Takashima N, Hayakawa M. Selective cerebral perfusion with mild hypothermic lower body circulatory arrest is safe for aortic arch surgery. Eur J Cardiothorac Surg 2013; 43(4): e94-8.

[20] Milewski RK, Pacini D, Moser GW, Moeller P, Cowie D, Szeto WY, et al. Retrograde and antegrade cerebral perfusion: Results in short elective arch reconstructive times. Ann Thorac Surg 2010; 89(5): 1448-57.

[21] Pasic M, Schubel J, Bauer M, Yankah C, Kuppe H, Weng YG, Hetzer R. Cannulation of the right axillary artery for surgery of acute type A aortic dissection. Eur J Cardiothorac Surg 2003; 24(2): 231-5.

[22] Moizumi Y, Motoyoshi N, Sakuma K, Yoshida S. Axillary artery cannulation improves operative results for acute type a aortic dissection. Ann Thorac Surg 2005; 80(1): 77-83.

[23] Suehiro S, Shimizu K, Ito M, Nakata H, Akeho K, Oda T. Recovery from very long aortic cross-clamping in redo complex aortic surgery. Gen Thorac Cardiovasc Surg 2017 Aug 29; doi: 101007/s11748-017-0823-8 [Epub ahead of print].

[24] Kuh JH, Kim JH, Choi JB. Myocardial protection and hemostasis in aortic root surgery: In resident training. Ann Thorac Surg 2016; 102(4): 1412

[25] Lee H, Cho YH, Sung K, Kim WS, Park KH, Park PW, Lee YT. Clinical outcomes of valve-sparing root replacement in acute type A aortic dissection. Scand Cardiovasc J 2015; 49(6): 331-6.

[26] Chiappini B, Schepens M, Tan E, Dell' Amore A, Morshuis W, Dossche K, et al. Early and late outcomes of acute type A aortic dissection: Analysis of risk factors in 487 consecutive patients. Eur Heart J 2005; 26(2): 180-6.

[27] Tan ME, Morshuis WJ, Dossche KM, Kelder JC, Waanders FG, Schepens MA. Long-term results after 27 years of surgical treatment of acute type a aortic dissection. Ann Thorac Surg 2005; 80(2): 523-9. 